\title{
Crossed Modules of Monoids I: Relative Categories
}

\author{
Gabriella Böhm ${ }^{1}$
}

Received: 14 March 2018 / Accepted: 19 June 2019 / Published online: 10 August 2019

(c) The Author(s) 2019

\begin{abstract}
This is the first part of a series of three strongly related papers in which three equivalent structures are studied:

- internal categories in categories of monoids, defined in terms of pullbacks relative to a chosen class of spans

- crossed modules of monoids relative to this class of spans

- simplicial monoids of so-called Moore length 1 relative to this class of spans.
\end{abstract}

The most important examples of monoids that are covered are small categories (treated as monoids in categories of spans) and bimonoids in symmetric monoidal categories (regarded as monoids in categories of comonoids). In this first part the theory of relative pullbacks is worked out leading to the definition of a relative category.

Keywords Relative pullback · Internal category · Crossed module

\section{Introduction}

Loosely speaking, a crossed module of a group [44] looks like a normal subgroup but it needs not be an inclusion in general. Its significance stems from its relation to various structures. A strict 2-group means an internal category in the category of groups. Its internal nerve is a simplicial group whose Moore complex is concentrated in degrees 1 and 2. The Moore complex is the corresponding crossed module. These constructions establish, in fact, equivalences between these three notions. Via the above links, crossed modules found diverse applications: in combinatorial homotopy, differential geometry, the theory of classifying spaces, in non-abelian cohomology and even in (mathematical) physics, in topological and homotopical quantum field theories [2,4-6,9-11,15,29,38-41,43-46]. Nice surveys can be found in [33,37].

A proof of the equivalence between crossed modules and strict 2-groups can be found in [13], where it is referred also to an unpublished proof [16]. Based on purely category

Communicated by George Janelidze.

Gabriella Böhm

bohm.gabriella@wigner.mta.hu

1 Wigner Research Centre for Physics, 114, P.O.B. 49, Budapest 1525, Hungary 
theoretical arguments, using the semi-abelian structure of the category of groups, in [26] George Janelidze gave another concise and highly elegant proof. An extensive analysis in the semi-abelian context was carried out in [42]. Semi-abelian categories include, in addition to the category of groups, also the category of Lie algebras, categories of varieties of $\Omega$-groups in the sense of [25], the category of Heyting semi-lattices, the dual of the category of pointed sets and much more. Thus by working in an arbitrary semi-abelian category, not only a more transparent proof is obtained, but also a much wider generality, also unifying earlier results in $[27,35]$.

Groups can be thought of as the Hopf monoids in the cartesian monoidal category of sets. Indeed, in any monoidal category one can discuss monoids (i.e. objects equipped with an associative and unital multiplication). Ordinary monoids are re-covered as monoids in the cartesian monoidal category of sets. Dually, one can define comonoids in arbitrary monoidal categories as monoids in the opposite category. In cartesian monoidal categories every object has a unique comonoid structure so this gives nothing interesting in the category of sets. Whenever a monoidal category is braided as well-that is, there is a natural isomorphism allowing to switch the order of the factors in the monoidal product - both monoids and comonoids of this monoidal category constitute monoidal categories. Using this fact, one can define bimonoids as monoids in the category of comonoids; equivalently, as comonoids in the category of monoids. Again, if the monoidal structure is cartesian (e.g. in the category of sets) this gives nothing new: bimonoids coincide with monoids. Hopf monoids in braided monoidal categories are distinguished bimonoids for which a canonical morphism is invertible. Hopf monoids in the category of sets are precisely the groups. Hopf monoids have been studied most intensively in the category of vector spaces where they are known as Hopf algebras.

Motivated by various applications, some research on crossed modules of Hopf algebras $[19,31]$ and of more general Hopf monoids $[1,20]$ began. In these papers, crossed modules of Hopf monoids were related to category-like objects in the category of Hopf monoids. Most recently, after making this paper public in the arXiv, in [23] the category of cocommutative Hopf algebras was proven to be semi-abelian; and therefore from Janelidze's theorem [26] the equivalence between internal categories and crossed modules of cocommutative Hopf algebras was derived. In [18] crossed modules over cocommutative Hopf algebras were related to cocommutative simplicial Hopf algebras with length 2 Moore complex (using arguments based on direct computation).

While Janelidze's approach in [26] via semi-abelian categories gives a very short proof and a very clear explanation of the equivalence between internal categories and crossed modules, it is not directly applicable to categories of Hopf monoids in arbitrary braided monoidal categories. While groups constitute a semi-abelian category, general Hopf monoids do not (their category is not even protomodular by [21]; see however [22,23]). In order to obtain a theory which is conceptually as clear as [26], but has a wider application, in the current series of papers we develop a theory dealing with monoids in general-not necessarily cartesianmonoidal categories. In this way we recover the following classes of examples:

- The classical notion of crossed modules of groups was generalized to monoids in [34]. Our theory covers it if applied to the cartesian monoidal category of sets. (The generalization in [32], however, seems to be beyond our scope.)

- In the paper [12] one can find the definition of crossed modules of groupoids, which is generalized to any categories in a straightforward way. Regarding small categories as monoids in categories of spans, in our theory we re-obtain the crossed modules of small categories as a particular case. 
- In [20] one can find the definition of crossed modules of Hopf monoids in symmetric monoidal categories, which is again smoothly generalized to bimonoids. Regarding bimonoids as monoids in categories of comonoids, in our theory we re-obtain the crossed modules of bimonoids (so in particular of ordinary monoids in the category of sets) as a particular case. Placing the results of [20] in our more general framework, we also find a conceptual reason why they only hold in a symmetric monoidal category, what obstructs the generalization to an arbitrary braiding.

Internal categories can be defined in arbitrary categories possibly admitting no pullbacks [30, Chapter III Section 6]. Dealing with such a category C, one may use the Yoneda embedding of $C$ into the presheaf category $C^{*}$ (of functors $C^{o p} \rightarrow$ set); and consider a category object in $C^{*}$ whose object of objects and object of morphisms are representable functors. This classical notion, however, turns out not to be useful for our purposes. Hence in carrying out our programme, the first question to understand is what to mean by an internal category in categories where arbitrary pullbacks may not exist (note the lack of pullbacks in categories of comonoids of our main interest). Resolving this problem, in this first part of the series we propose some 'admissibility' axioms on a class of spans and define pullbacks relative to such a class $\mathcal{S}$. Assuming that relative pullbacks of those cospans whose 'legs are in $\mathcal{S}$ ' -a terminology to be made precise later in Definition 2.10 - exist, as they do in the examples in our mind, we obtain a monoidal category whose objects are the spans with their legs in $\mathcal{S}$. An $\mathcal{S}$-relative category is meant then to be a monoid therein.

Working in a monoidal category $C$, we may require the compatibility of our admissible class $\mathcal{S}$ of spans with the monoidal structure. With this compatibility at hand, $\mathcal{S}$ induces an admissible class of spans in the category of monoids in C; hence relative categories in the category of monoids are available. In Part II of this series [7] their category is shown to be equivalent to the category of relative crossed modules of monoids in a suitable sense; and in Part III [8] to the category of relative simplicial monoids of so-called Moore length 1.

Extending the picture on internal categories and crossed modules (of groups) recalled above, suitable nerves of Cat ${ }^{n}$-groups (i.e. $n$-fold categories in the category of groups) are simplicial groups whose Moore complex is concentrated in degrees up-to $n+1$ and these Moore complexes are known as $n$-crossed modules. Again, these correspondences are in fact equivalences [14,36]. These equivalent viewpoints are both of conceptual and practical use: each of them gives a different insight and interpretation of the same thing; and they provide the possibility for finding the (sometimes technically) smoothest approach in the applications $[14,17,24,28,36]$. We believe that our methods should be suitable to obtain an analogous theory of higher relative categories and crossed modules of monoids what we plan to discuss elsewhere.

\section{Preliminaries on Monoids in Monoidal Categories}

In this preliminary section we recall—without, or with very sketchy proofs-some known facts about monoids that will play important roles in our later constructions; in particular Part II [7]. Throughout the section $M$ denotes a monoidal category whose monoidal unit is $I$ and the monoidal product is denoted by juxtaposition. For the monoidal product of $n$ copies of the same object $A$ also the power notation $A^{n}$ is used. The monoidal structure is not assumed to be strict but the associativity and unit coherence isomorphisms are not explicitly denoted. Whenever $M$ is assumed to be braided monoidal, its braiding will be denoted by $c$. Composition of morphisms $f: A \rightarrow B$ and $g: B \rightarrow C$ is denoted by $g \cdot f: A \rightarrow C$ and identity morphisms are denoted by 1 . 
Definition 1.1 A monoid in $\mathrm{M}$ consists of an object $A$ together with a multiplication morphism $A^{2} \stackrel{m}{\rightarrow} A$ and a unit morphism $I \stackrel{u}{\rightarrow} A$ such that the associativity condition $m \cdot m 1=m \cdot 1 m$ and the unit conditions $m \cdot u 1=1=m \cdot 1 u$ hold (note the omitted coherence isomorphisms). A monoid morphism is a morphism $A \stackrel{f}{\rightarrow} A^{\prime}$ for which $f \cdot m=m^{\prime} \cdot f f$ and $f \cdot u=u^{\prime}$.

Lemma 1.2 Two monoid morphisms $A-f>C<g-B$ in $M$ are jointly epimorphic morphisms of monoids whenever the induced morphism

$$
q:=A B \stackrel{f g}{\longrightarrow} C^{2} \stackrel{m}{\longrightarrow} C
$$

is an epimorphism in $M$.

Proof If $x \cdot f=y \cdot f$ and $x \cdot g=y \cdot g$ for some parallel monoid morphisms $x$ and $y$, then also $x \cdot q=y \cdot q$.

Definition 1.3 A distributive law in $\mathrm{M}$ consists of two monoids $A$ and $B$ together with a morphism $B A \stackrel{x}{\rightarrow} A B$ such that the following identities (using the notation $m$ for both multiplications and $u$ for both units) hold.

$$
\begin{array}{ll}
x \cdot m 1=1 m \cdot x 1 \cdot 1 x & x \cdot u 1=1 u \\
x \cdot 1 m=m 1 \cdot 1 x \cdot x 1 & x \cdot 1 u=u 1
\end{array}
$$

Lemma 1.4 For any distributive law $B A \stackrel{x}{\rightarrow} A B$, there is an induced monoid structure on $A B$ with the unit $I \stackrel{u u}{\longrightarrow} A B$ and the multiplication $(A B)^{2} \stackrel{1 x 1}{\longrightarrow} A^{2} B^{2} \stackrel{m m}{\longrightarrow} A B$. For this monoid both $A \stackrel{1 u}{\longrightarrow} A B$ and $B \stackrel{u 1}{\longrightarrow} A B$ are monoid morphisms.

Lemma 1.5 Consider monoid morphisms $A-f>C<g-B$ such that the induced morphism $q$ of (1.1) is invertible. Then the unique monoid structure on $A B$ for which $q$ is a monoid morphism is induced by the distributive law

$$
B A \stackrel{g f}{\longrightarrow} C^{2} \stackrel{m}{\longrightarrow} C \stackrel{q^{-1}}{\longrightarrow} A B .
$$

Lemma 1.6 For a distributive law $B A \stackrel{x}{\rightarrow} A B$ and a monoid $C$, there is a bijective correspondence between the following data.

(i) monoid morphisms $A B \stackrel{c}{\rightarrow} C$ (where the monoid structure of $A B$ is induced by $x$ )

(ii) pairs of monoid morphisms $(A \stackrel{a}{\rightarrow} C, B \stackrel{b}{\rightarrow} C$ ) such that $m \cdot a b \cdot x=m \cdot b a$.

Proof A monoid morphism $c$ in part (i) is sent to the pair $(c \cdot 1 u, c \cdot u 1)$. Conversely, a pair $(a, b)$ in part (ii) is sent to $m \cdot a b$.

Corollary 1.7 Consider monoid morphisms $A-f>C<g-B$ such that the induced morphism q of (1.1) is invertible. For any monoid $D$, there are mutually inverse bijections below. 
(i) The map sending a monoid morphism $C \stackrel{c}{\rightarrow} D$ to the pair of monoid morphisms $(c \cdot f, c \cdot g)$ rendering commutative the following diagram.



(ii) The map sending a pair of monoid morphisms $(A \stackrel{a}{\rightarrow} D, B \stackrel{b}{\rightarrow} D$ ), satisfying $m \cdot a b$. $q^{-1} \cdot m \cdot g f=m \cdot b a$, to the monoid morphism $C \stackrel{q^{-1}}{\longrightarrow} A B \stackrel{a b}{\longrightarrow} D^{2} \stackrel{m}{\longrightarrow} D$.

Proof The top row of the diagram of part (i) is a distributive law by Lemma 1.5. Hence Lemma 1.6 yields a bijection between the pairs of monoid morphisms as in (ii) and the monoid morphisms $A B \rightarrow D$. Composition with the monoid isomorphism $q^{-1}$ yields then the stated bijection with the monoid morphisms $C \rightarrow D$.

\section{Admissible Classes of Spans}

We are interested in categories-like the categories of comonoids in symmetric monoidal categories, see the Introduction - in which general pullbacks may not exist. Instead, we will assume the existence of certain relative pullbacks with respect to some distinguished class of spans. By this motivation in this section we investigate the expected properties of such a class.

Definition 2.1 A class $\mathcal{S}$ of spans in any category $\mathrm{C}$ is said to be admissible if it satisfies the following two conditions.

(POST) If $X \stackrel{f}{\longleftarrow} A \stackrel{g}{\rightarrow} Y \in \mathcal{S}$ then $X^{\prime} \stackrel{f^{\prime}}{\longleftarrow} X \stackrel{f}{\longleftarrow} A \stackrel{g}{\longrightarrow} Y \stackrel{g^{\prime}}{\longrightarrow} Y^{\prime} \in \mathcal{S}$ too, for any morphisms $X \stackrel{f^{\prime}}{\rightarrow} X^{\prime}$ and $Y \stackrel{g^{\prime}}{\rightarrow} Y^{\prime}$.

(PRE) If $X \stackrel{f}{\leftarrow} A \stackrel{g}{\longrightarrow} Y \in \mathcal{S}$ then $X \stackrel{f}{\leftarrow} A \stackrel{h}{\leftarrow} B \stackrel{h}{\longrightarrow} A \stackrel{g}{\longrightarrow} Y \in \mathcal{S}$ too, for any morphism $B \stackrel{h}{\rightarrow} A$.

Example 2.2 The class of all spans in a category is clearly admissible.

Example 2.3 For a monoidal category $\mathrm{M}$, let $\mathrm{C}$ be the category of comonoids in $\mathrm{M}$ (that is, the category of monoids in the monoidal category $M^{\text {rev }}$ with the opposite composition). Assume that $\mathrm{M}$ is braided monoidal (with braiding $c$ ). Then $\mathrm{C}$ inherits the monoidal structure of $\mathrm{M}$ : the monoidal unit $I$ is a trivial comonoid with comultiplication $I \cong I^{2}$ provided by the unit isomorphisms, and the monoidal product $A C$ of any comonoids $A$ and $C$ is a comonoid via the comultiplication $A C \stackrel{\delta \delta}{\rightarrow} A^{2} C^{2} \stackrel{1 c 1}{\rightarrow}(A C)^{2}$ induced by the comultiplications $A \stackrel{\delta}{\rightarrow} A^{2}$ and $C \stackrel{\delta}{\rightarrow} C^{2}$. 
Define $\mathcal{S}$ to contain precisely those spans $X \stackrel{f}{\leftarrow} A \stackrel{g}{\rightarrow} Y$ in $C$ for which the composite morphism $A \stackrel{\delta}{\rightarrow} A^{2} \stackrel{f g}{\rightarrow} X Y$ is a comonoid morphism; equivalently, the equality $c \cdot f g \cdot \delta=$ $g f \cdot \delta$ holds.

For any comonoid morphisms $f^{\prime}$ and $g^{\prime}$ of respective domains $X$ and $Y$, the monoidal product $f^{\prime} g^{\prime}$ is a comonoid morphism. Then so is $f^{\prime} g^{\prime} \cdot f g \cdot \delta$ for any $X \stackrel{f}{\leftarrow} A \stackrel{g}{\longrightarrow} Y \in \mathcal{S}$, so that condition (POST) is satisfied.

On the other hand, for any comonoid morphism $h$ of codomain $A, f g \cdot \delta \cdot h=f g \cdot h h \cdot \delta$ is a comonoid morphism so also (PRE) holds.

The current example can be considered in the particular situation when $M$ is a cartesian monoidal (so symmetric monoidal) category. Then every object has a unique comonoid structure; that is, $\mathrm{C}$ and $\mathrm{M}$ are isomorphic. In particular, every comonoid is cocommutative (that is, the comultiplication $\delta$ and the symmetry $c$ satisfy $c \cdot \delta=\delta$ ). Then the class $\mathcal{S}$ of spans above is the class of all spans in $C \cong M$.

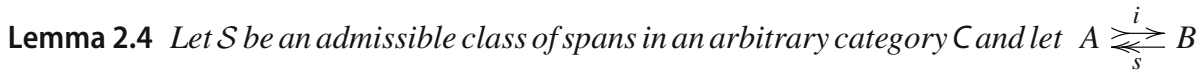
be a split epimorphism in $C$ (that is, $s \cdot i=1)$.

(1) The following assertions are equivalent.

(a) $B=B=B \in \mathcal{S}$.

(b) $X \stackrel{f}{\leftarrow} B \stackrel{g}{\longrightarrow} Y \in \mathcal{S}$ for any morphisms $f$ and $g$ of domain $B$.

(c) $A \stackrel{i}{\leftarrow} B=B \in \mathcal{S}$.

(2) The equivalent assertions of part (1) hold whenever $A=A \stackrel{s}{\rightarrow} B \in \mathcal{S}$.

Proof Assertion (a) of part (1) implies (b) by condition (POST) on $\mathcal{S}$, post-composing by $f$ on the left and by $g$ on the right. Assertion (b) trivially implies (c). Finally, (c) implies (a) by (POST), post-composing on the left by $s$ and using $s \cdot i=1$. The condition in part (2) implies (c) of part (1) by (PRE), pre-composing by $i$ and using $s \cdot i=1$ again.

Definition 2.5 A class $\mathcal{S}$ of spans in a monoidal category M is said to be monoidal if it satisfies the following two conditions.

(UNITAL)

For any morphisms $f$ and $g$ whose domain is the monoidal unit $I$,

$$
X \stackrel{f}{\leftarrow} I \stackrel{g}{\longrightarrow} Y \in \mathcal{S} \text {. }
$$

(MULTIPLICATIVE) If both $X \stackrel{f}{\leftarrow} A \stackrel{g}{\longrightarrow} Y \in \mathcal{S}$ and $X^{\prime} \stackrel{f^{\prime}}{\leftarrow} A^{\prime} \stackrel{g^{\prime}}{\longrightarrow} Y^{\prime} \in \mathcal{S}$ then also

$$
X X^{\prime} \stackrel{f f^{\prime}}{\leftarrow} A A^{\prime} \stackrel{g g^{\prime}}{\longrightarrow} Y Y^{\prime} \in \mathcal{S} \text {. }
$$

A class of spans satisfying (POST) is unital if and only if $I=I=I \in \mathcal{S}$.

Example 2.6 The class of all spans in a monoidal category is clearly monoidal.

Example 2.7 For a braided monoidal category $\mathrm{M}$ (with braiding $c$ ) let $\mathrm{C}$ be the category of comonoids in $\mathrm{M}$. It is monoidal via the monoidal product of $\mathrm{M}$, see Example 2.3. Below we show that the class $\mathcal{S}$ in Example 2.3 of spans in $C$ is monoidal whenever the symmetry is a braiding; that is, $c^{-1}=c$. (This explains in a conceptual way why in [20] it is dealt only with symmetric monoidal categories not with arbitrary braidings.) 
By the coherence of the braiding, the comultiplication $\delta$ of $I$ satisfies $c \cdot \delta=\delta$. Then $I=I=I \in \mathcal{S}$, and so the unitality of $\mathcal{S}$ follows by its property (POST), see Example 2.3 .

For any $X \stackrel{f}{\longleftarrow} A \stackrel{g}{\longrightarrow} Y \in \mathcal{S}$ and $X^{\prime} \stackrel{f^{\prime}}{\longleftarrow} A^{\prime} \stackrel{g^{\prime}}{\longrightarrow} Y^{\prime} \in \mathcal{S}$ the following diagram commutes.

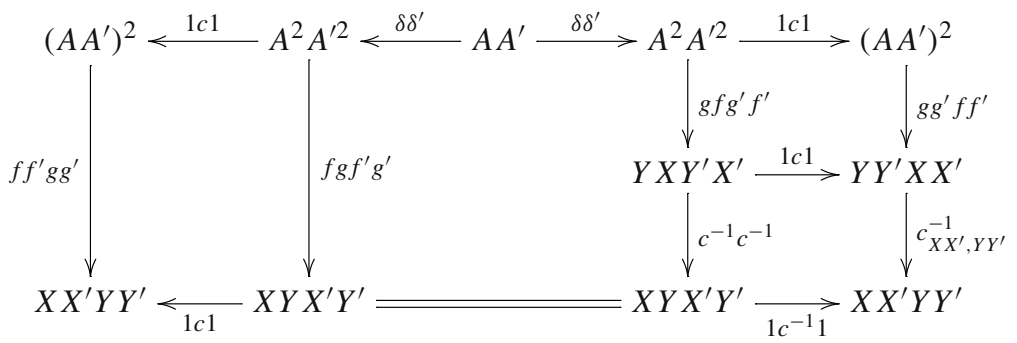

If $c$ is a symmetry, then the arrows of the bottom row are equal isomorphisms proving the equality of the top-left and the top-right paths; that is, $X X^{\prime} \stackrel{f f^{\prime}}{\longleftarrow} A A^{\prime} \stackrel{g g^{\prime}}{\longrightarrow} Y Y^{\prime} \in \mathcal{S}$.

Example 2.8 Consider any class $\mathcal{S}^{\prime}$ of spans in an arbitrary category $C^{\prime}$. For any functor $U: C \rightarrow C^{\prime}$ define the class $\mathcal{S}$ which contains precisely those spans in $\mathrm{C}$ whose image belongs to $\mathcal{S}^{\prime}$.

(1) If $\mathcal{S}^{\prime}$ is admissible then so is $\mathcal{S}$.

(2) Assume that $C$ and $C^{\prime}$ are monoidal categories and $U$ is a strict monoidal functor. If $\mathcal{S}^{\prime}$ is monoidal then so is $\mathcal{S}$.

For (1) note that $X \stackrel{f}{\leftarrow} A \stackrel{g}{\longrightarrow} Y \in \mathcal{S}$ if and only if $U X \stackrel{U f}{<} U A \stackrel{U g}{\longrightarrow} U Y \in \mathcal{S}^{\prime}$. If this is the case, then by property (POST) of $\mathcal{S}^{\prime}$, also $U X^{\prime} \stackrel{U f^{\prime}}{\leftarrow} U X \stackrel{U f}{\leftarrow} U A \stackrel{U g}{\longrightarrow} U Y \stackrel{U g^{\prime}}{\longrightarrow} U Y^{\prime} \in \mathcal{S}^{\prime}$ for all morphisms $f^{\prime}$ and $g^{\prime}$ in $C$ with respective domains $X$ and $Y$. By definition this is equivalent to $X^{\prime} \stackrel{f^{\prime}}{\leftarrow} X \stackrel{f}{\leftarrow} A \stackrel{g}{\longrightarrow} Y \stackrel{g^{\prime}}{\rightarrow} Y^{\prime} \in \mathcal{S}$ proving property (POST) of $\mathcal{S}$. Analogous reasoning applies to property (PRE).

For (2) observe that for any span $X \stackrel{f}{\leftarrow} I \stackrel{g}{\rightarrow} Y$ in $\mathcal{C}, U X \stackrel{U f}{\leftarrow} U I=I^{\prime} \stackrel{U g}{\longrightarrow} U Y \in \mathcal{S}^{\prime}$ by the unitality of $\mathcal{S}^{\prime}$. Then $X \stackrel{f}{\leftarrow} I \stackrel{g}{\rightarrow} Y \in \mathcal{S}$ by definition so that $\mathcal{S}$ is unital.

For $X \stackrel{f}{<} A \stackrel{g}{\longrightarrow} Y \in \mathcal{S}$ and $X^{\prime} \stackrel{f^{\prime}}{<} A^{\prime} \stackrel{g^{\prime}}{\rightarrow} Y^{\prime} \in \mathcal{S}, U X \stackrel{U f}{\leftarrow} U A \stackrel{U g}{\longrightarrow} U Y \in \mathcal{S}^{\prime}$ and $U X^{\prime} \stackrel{U f^{\prime}}{\leftarrow} U A^{\prime} \stackrel{U g^{\prime}}{\longrightarrow} U Y^{\prime} \in \mathcal{S}^{\prime}$ by definition. Then by the multiplicativity of $\mathcal{S}^{\prime}$, also

$$
\begin{aligned}
& U\left(f f^{\prime}\right)=(U f)\left(U f^{\prime}\right) \quad U\left(g g^{\prime}\right)=(U g)\left(U g^{\prime}\right) \\
& U\left(X X^{\prime}\right)=(U X)\left(U X^{\prime}\right) \longleftarrow U\left(A A^{\prime}\right)=U(A) U\left(A^{\prime}\right) \longrightarrow U\left(Y Y^{\prime}\right)=(U Y)\left(U Y^{\prime}\right) \in \mathcal{S}^{\prime} .
\end{aligned}
$$

By definition this is equivalent to $X X^{\prime} \stackrel{f f^{\prime}}{\longleftarrow} A A^{\prime} \stackrel{g g^{\prime}}{\longrightarrow} Y Y^{\prime} \in \mathcal{S}$, proving the multiplicativity of $\mathcal{S}$.

Example 2.9 As a particular instance of Example 2.8, consider a monoidal category $M$ and a class $\mathcal{S}^{\prime}$ of spans in $\mathrm{M}$. Take $\mathrm{C}$ to be the category of monoids in $\mathrm{M}$ and $\mathcal{S}$ to be the class containing precisely those spans in $\mathrm{C}$ whose image under the forgetful functor $U: \mathrm{C} \rightarrow \mathrm{M}$ belongs to $\mathcal{S}^{\prime}$. From Example 2.8 we infer the following. 
(1) If $\mathcal{S}^{\prime}$ is admissible then so is $\mathcal{S}$.

(2) Assume that $\mathrm{M}$ is a braided monoidal category (so that also $\mathrm{C}$ is monoidal and $U$ is strict monoidal). If $\mathcal{S}^{\prime}$ is monoidal then so is $\mathcal{S}$.

Definition 2.10 For any class $\mathcal{S}$ of spans in some category $\mathrm{C}$ we say that a cospan $A \stackrel{f}{\longrightarrow} B \stackrel{g}{\leftarrow} C$ has legs in $\mathcal{S}$ if $A=A \stackrel{f}{\longrightarrow} B$ and $B \stackrel{g}{\leftarrow} C=C$ belong to $\mathcal{S}$.

A span $B \stackrel{g}{\leftarrow} A \stackrel{f}{\longrightarrow} B$ (with equal objects at the left and the right) is said to have its legs in $\mathcal{S}$ if the cospan $A \stackrel{f}{\longrightarrow} B \stackrel{g}{\leftarrow} A$ has legs in $\mathcal{S}$.

\section{Relative Pullbacks}

Definition 3.1 Consider an admissible class $\mathcal{S}$ of spans in an arbitrary category C. For some morphisms $A \stackrel{a}{\rightarrow} B \stackrel{c}{\leftarrow} C$ in $C$ the relative pullback with respect to $\mathcal{S}$-if it exists-is a span $A \stackrel{p_{A}}{<} A \square_{B} C \stackrel{p_{C}}{\longrightarrow} C$ belonging to $\mathcal{S}$ such that the following properties hold (see diagram (3.1) below).

(i) $a \cdot p_{A}=c \cdot p_{C}$

(ii) Universality: for any $A \stackrel{f}{\leftarrow} X \stackrel{g}{\rightarrow} C \in \mathcal{S}$ such that $a \cdot f=c \cdot g$, there is a unique morphism $X \stackrel{h}{\rightarrow} A \square_{B} C$ in $C$ which satisfies $p_{A} \cdot h=f$ and $p_{C} \cdot h=g$.

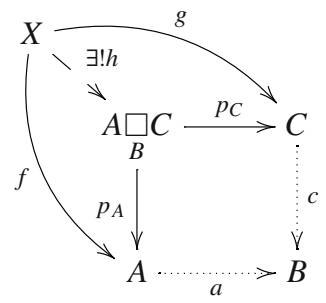

(iii) Reflection: if both $A \stackrel{p_{A}}{\leftarrow} A \square_{B} C \stackrel{f}{\leftarrow} D \stackrel{g}{\longrightarrow} E$ and $C \stackrel{p_{C}}{<} A \underset{B}{\leftarrow} C \stackrel{f}{\longleftarrow} D \stackrel{g}{\longrightarrow} E$ belong to $\mathcal{S}$, then also $A \underset{B}{\square} C \stackrel{f}{\leftarrow} D \stackrel{g}{\rightarrow} E$ belongs to $\mathcal{S}$; and symmetrically, if both $E \stackrel{g}{\leftarrow} D \stackrel{f}{\rightarrow} A \square_{B} C \stackrel{p_{A}}{\longrightarrow} A$ and $E \stackrel{g}{\leftarrow} D \stackrel{f}{\rightarrow} A \square_{B} C \stackrel{p_{C}}{\longrightarrow} C$ belong to $\mathcal{S}$, then also $E \stackrel{g}{\leftarrow} D \stackrel{f}{\rightarrow} A \underset{B}{\longrightarrow} C$ belongs to $\mathcal{S}$.

By property (PRE) of $\mathcal{S}$, part (ii) of Definition 3.1 implies that the morphisms $A \stackrel{p_{A}}{\longleftarrow} A \underset{B}{\square} \stackrel{p_{C}}{\longrightarrow} C$ are jointly monic. Therefore the $\mathcal{S}$-relative pullback is unique up-to isomorphism whenever it exists.

Example 3.2 If $\mathcal{S}$ is the class of all pullbacks in some category $\mathrm{C}$, then $\mathcal{S}$-relative pullbacks are just usual pullbacks. 
Example 3.3 As in Example 2.3, let $\mathrm{C}$ be the category of comonoids in a monoidal category $M$. Assume that $M$ has equalizers which are preserved by taking the monoidal product with any object on either side. Then in $C$ any parallel morphisms $A \underset{g}{\stackrel{f}{\rightrightarrows}} B$ have an equalizer; computed as the equalizer

$$
E \stackrel{j}{\longrightarrow} A \frac{\widehat{f}:=1 f 1 \cdot \delta 1 \cdot \delta}{\widehat{g}:=1 g 1 \cdot \delta 1 \cdot \delta} \rightarrow A B A
$$

in $\mathrm{M}$, where $\delta$ stands for the comultiplication of $A$. (In [3, Lemma 1.1.3 (4)] the analogous form of the equalizers in the category of Hopf algebras over a field was verified.) Clearly, any comonoid morphism of codomain $A$ equalizes $f$ and $g$ if and only if it equalizes $\widehat{f}$ and $\widehat{g}$. So in order to prove that $j: E \rightarrow A$ is the equalizer of $f$ and $g$ in C, we need to equip $E$ with a comonoid structure so that $j$ becomes a comonoid (mono)morphism.

The counit is $E \stackrel{j}{\rightarrow} A \stackrel{\varepsilon}{\rightarrow} I$, where $\varepsilon$ stands for the counit of $A$. The comultiplication is constructed in two steps. First the universality of the equalizer in $\mathrm{M}$ in the bottom row of the first serially commutative diagram below is used to construct an auxiliary morphism $\delta_{r}$; and then the comultiplication $\delta$ is constructed using the universality of the equalizer in $M$ in the bottom row of the second serially commutative diagram in
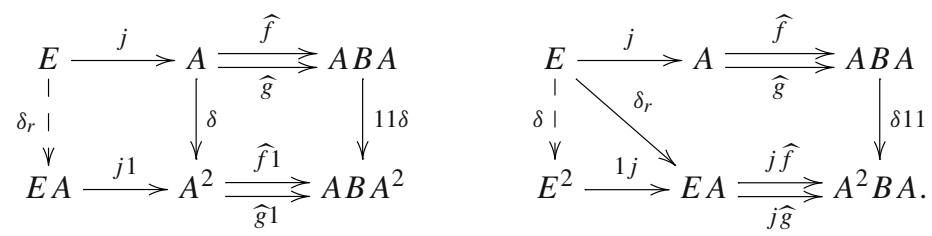

Assume furthermore that $\mathrm{M}$ is a braided monoidal category so that $\mathrm{C}$ inherits the monoidal structure of M (cf. Example 2.3). Any comonoid morphisms $A \stackrel{f}{\longrightarrow} B \stackrel{g}{\leftarrow} C$ induce comonoid morphisms $A C \underset{\varepsilon g}{\stackrel{f \varepsilon}{\rightleftarrows}} B$ (where $\varepsilon$ stands for both counits of $A$ and $C$ ). So we can take their equalizer

$$
A \square C \stackrel{j}{\longrightarrow} A C \underset{\varepsilon g}{\stackrel{f \varepsilon}{\rightleftarrows}} B
$$

in C. Below we claim that it gives in fact the pullback

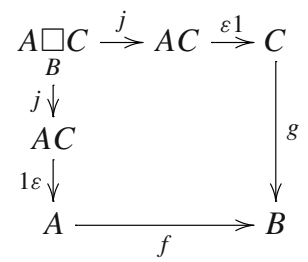

relative to the admissible class $\mathcal{S}$ in Example 2.3 of spans in C.

The square of (3.3) commutes since (3.2) is a fork. The span $A \stackrel{1 \varepsilon \cdot j}{\longleftarrow} A \square C \stackrel{\varepsilon 1 \cdot j}{\longrightarrow} C$ belongs to $\mathcal{S}$ since $1 \varepsilon \varepsilon 1 \cdot j j \cdot \delta=j$ is a comonoid morphism by construction. In order to 
check the universality of (3.3), take a span $A \stackrel{k}{\leftarrow} D \stackrel{l}{\longrightarrow} C$ in $\mathcal{S}$ for which $f \cdot k=g \cdot l$. Then $f \varepsilon \cdot k l \cdot \delta=f \cdot k=g \cdot l=\varepsilon g \cdot k l \cdot \delta$. Thus since $k l \cdot \delta$ is a comonoid morphism by assumption, a filler $h$ of the first diagram in

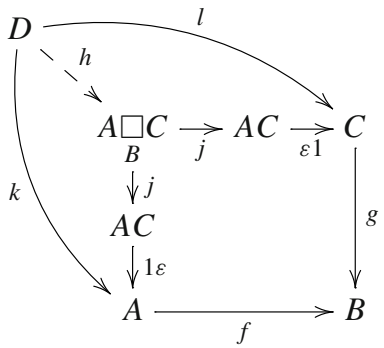

is constructed via the universality of the equalizer in $C$ in the bottom row of the second diagram. It is a comonoid morphism by construction. The uniqueness of a comonoid morphism $h$ rendering commutative the first diagram of (3.4) follows by the observation that any comonoid morphism $h$ making the first diagram commute, renders commutative also the second diagram of (3.4) by the commutativity of

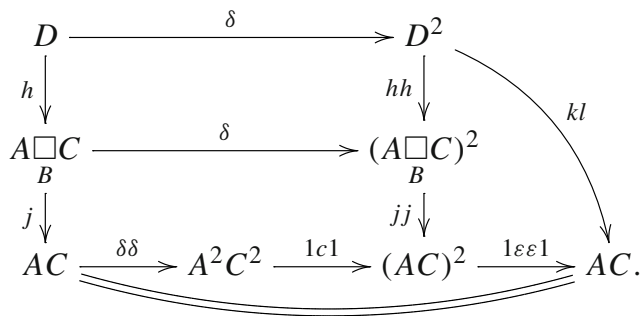

For the reflection property on the left, assume that

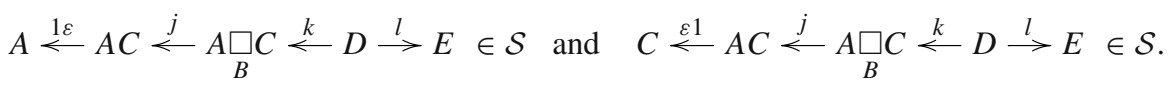

Then the diagram of Fig. 1 commutes (the region marked by (1) commutes by the first condition, and the region marked by (2) commutes by the second condition of (3.5)). Since the right column and the bottom row of the diagram of Fig. 1 are equal monomorphisms, this proves the equality of the left column and the top row; that is, $A \underset{B}{\square} \stackrel{k}{\leftarrow} D \stackrel{l}{\rightarrow} E \in \mathcal{S}$.

A symmetrical reasoning verifies the reflection property on the right.

Summarizing, we proved that the pullback relative to the class $\mathcal{S}$ in Example 2.3 of spans in $C$ exists for any comonoid morphisms $A \stackrel{f}{\longrightarrow} B \stackrel{g}{\leftarrow} C$. It is computed as the equalizer in $\mathrm{C}$ of the comonoid morphisms $A C \underset{\varepsilon g}{\stackrel{f \varepsilon}{\rightleftarrows}} B$.

Consider now comonoid morphisms $A \stackrel{f}{\longrightarrow} B \stackrel{g}{\leftarrow} C$ in a braided monoidal category M, such that $A=A \stackrel{f}{\rightarrow} B$ and $B \stackrel{g}{\leftarrow} C=C$ belong to the class $\mathcal{S}$ in Example 2.3 of spans in the category $C$ of comonoids in M. (In [20, Definition 5] this assumption occurs for 


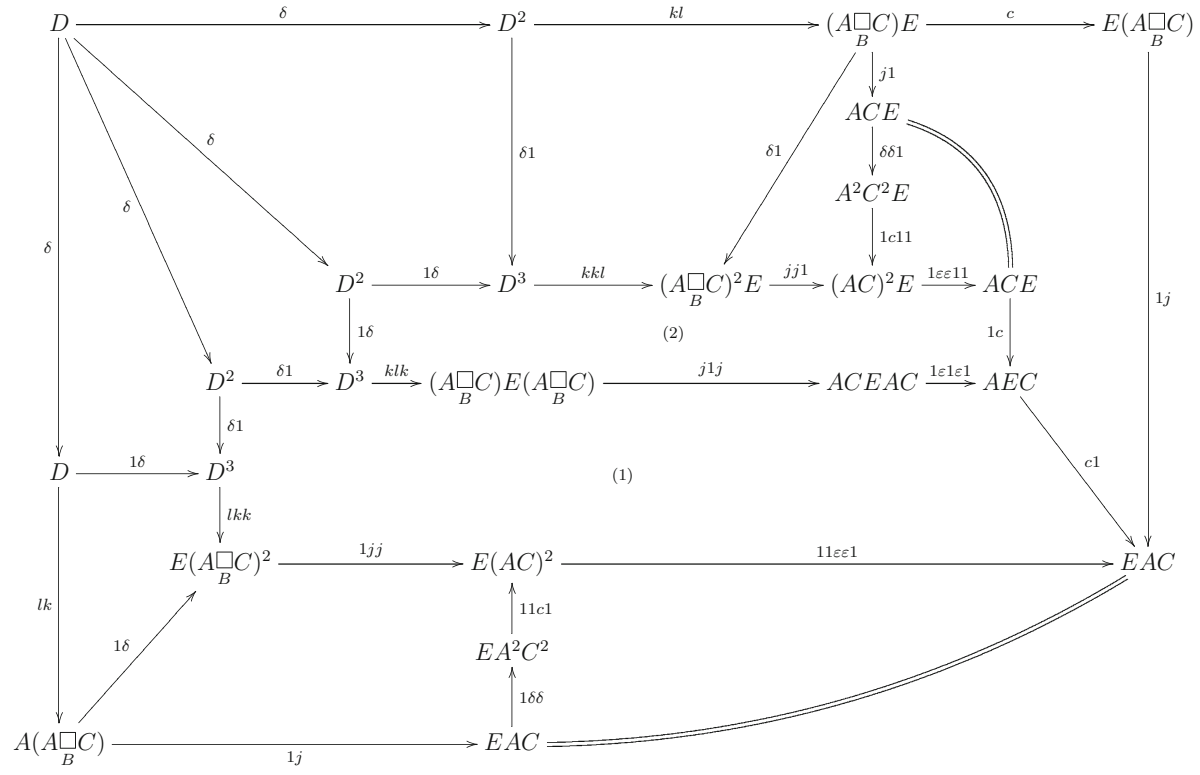

Fig. 1 Reflection property

morphisms of Hopf monoids in a symmetric monoidal category.) Then the $\mathcal{S}$-relative pullback (3.3) becomes isomorphic to the so-called cotensor product; defined as the equalizer

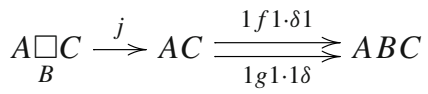

in $\mathrm{M}$, thanks to the serially commutative diagrams
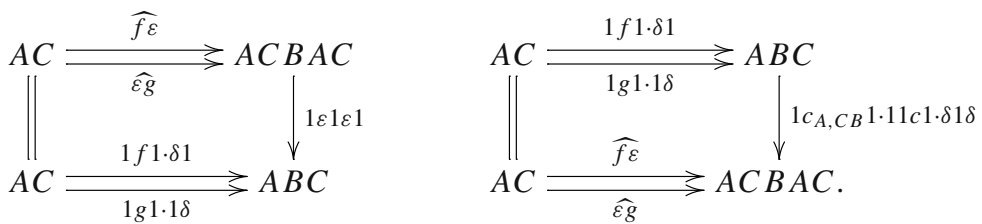

The current example can be considered in the particular situation when $\mathrm{M}$ is a cartesian symmetric monoidal category. Then the class $\mathcal{S}$ of spans in Example 2.3 is the class of all spans in $\mathrm{C} \cong \mathrm{M}$ and thus $\mathcal{S}$-relative pullbacks are just usual pullbacks; see Example 3.2.

Lemma 3.4 For any admissible class $\mathcal{S}$ of spans in an arbitrary category take an $\mathcal{S}$-relative pullback (3.1). The following assertions hold.

(1) If $A^{\prime} \stackrel{a}{\leftarrow} A=A \in \mathcal{S}$ then also $A^{\prime} \stackrel{a}{\leftarrow} A \stackrel{p_{A}}{\leftarrow} A \square_{B} C=A \square_{B} C \in \mathcal{S}$.

(2) If $C=C \stackrel{c}{\rightarrow} C^{\prime} \in \mathcal{S}$ then also $A \underset{B}{\square} C=A \underset{B}{ } C \stackrel{p_{C}}{\rightarrow} C \stackrel{c}{\rightarrow} C^{\prime} \in \mathcal{S}$. 
Proof We only prove part (1), part (2) follows analogously. By assumption the span $A^{\prime} \stackrel{a}{\leftarrow} A=A$ is in $\mathcal{S}$ hence by (PRE)

$$
A^{\prime} \stackrel{a}{\leftarrow} A \stackrel{p_{A}}{\longleftarrow} A \underset{B}{\square} \stackrel{p_{A}}{\longrightarrow} A \in \mathcal{S} .
$$

By construction $A \stackrel{p_{A}}{\leftarrow} A \underset{B}{\square} \stackrel{p_{C}}{\longrightarrow} C \in \mathcal{S}$. Then by (POST)

$$
A^{\prime} \stackrel{a}{\longleftarrow} A \stackrel{p_{A}}{\longleftarrow} A \underset{B}{\square} \stackrel{p_{C}}{\longrightarrow} C \in \mathcal{S} .
$$

By the reflection property of $A \square_{B} C$ the displayed properties imply the claim.

Proposition 3.5 Let $\mathcal{S}$ be an admissible class of spans in an arbitrary category. Consider $\mathcal{S}$-relative pullbacks
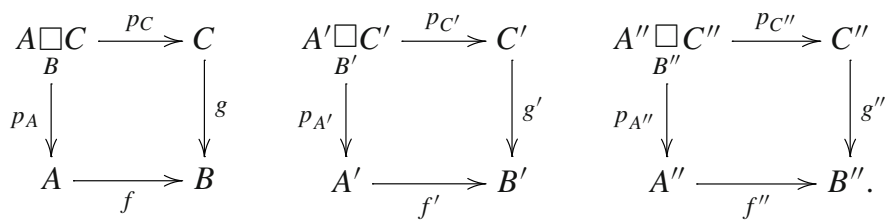

(1) For any morphisms $A \stackrel{a}{\longrightarrow} A^{\prime}, \quad B \stackrel{b}{\longrightarrow} B^{\prime}$ and $C \stackrel{c}{\longrightarrow} C^{\prime}$ such that $b \cdot f=f^{\prime} \cdot a$ and $b \cdot g=g^{\prime} \cdot c$, there is a unique morphism a $\square c$ rendering the following diagram commutative.

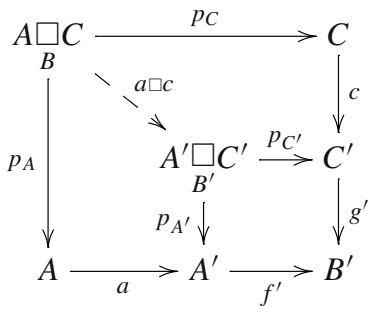

(2) The operation $\square$ of part (1) is functorial in the sense that for any further morphisms $A^{\prime} \stackrel{a^{\prime}}{\longrightarrow} A^{\prime \prime}, B^{\prime} \stackrel{b^{\prime}}{\longrightarrow} B^{\prime \prime}$ and $C^{\prime} \stackrel{c^{\prime}}{\longrightarrow} C^{\prime \prime}$ such that $b^{\prime} \cdot f^{\prime}=f^{\prime \prime} \cdot a^{\prime}$ and $b^{\prime} \cdot g^{\prime}=g^{\prime \prime} \cdot c^{\prime}$, the equality $\left(a^{\prime} \square c^{\prime}\right) \cdot(a \square c)=a^{\prime} \cdot a \square c^{\prime} \cdot c$ holds.

Proof (1) By construction $A \stackrel{p_{A}}{\leftarrow} A \underset{B}{\square} \stackrel{p_{C}}{\longrightarrow} C$ belongs to $\mathcal{S}$. Hence by property (POST), also $A^{\prime} \stackrel{a}{\gtrless} A \stackrel{p_{A}}{\gtrless} A \underset{B}{\square} C \stackrel{p_{C}}{\longrightarrow} C \stackrel{c}{\longrightarrow} C^{\prime}$ belongs to $\mathcal{S}$. Therefore the stated morphism $a \square c$ exists by the universality of the $\mathcal{S}$-relative pullback $A^{\prime} \square_{B^{\prime}} C^{\prime}$ and the commutativity of 


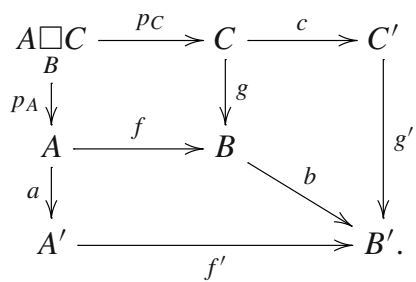

(2) Both morphisms $\left(a^{\prime} \square c^{\prime}\right) \cdot(a \square c)$ and $a^{\prime} \cdot a \square c^{\prime} \cdot c$ render commutative the same diagram

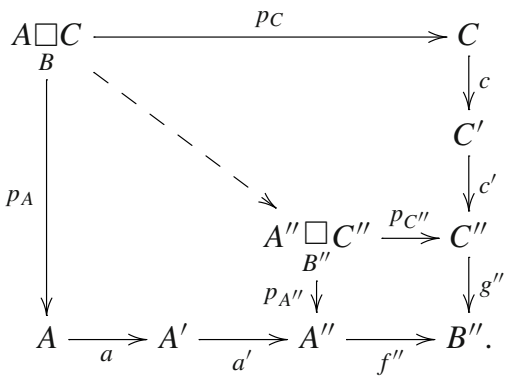

Hence they are equal by the universality of $A^{\prime \prime} \square_{B^{\prime \prime}} C^{\prime \prime}$.

Proposition 3.6 For any admissible class $\mathcal{S}$ of spans in an arbitrary category, the following assertions hold.

(1) If $A=A \stackrel{f}{\rightarrow} B \in \mathcal{S}$ then the first diagram below is an $\mathcal{S}$-relative pullback and if $B \stackrel{g}{\leftarrow} C=C \in \mathcal{S}$ then the second diagram is so.
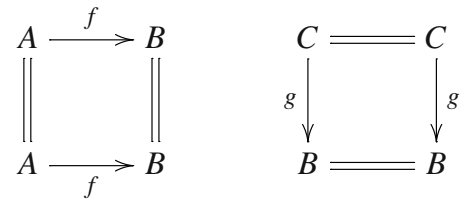

That is to say, $A \underset{B}{\square} B \stackrel{p_{A}}{\rightarrow} A$ and $B \underset{B}{\square} C \stackrel{p_{C}}{\rightarrow} C$ are isomorphisms.

(2) Consider morphisms $A \stackrel{f}{\longrightarrow} B \stackrel{g}{\leftarrow} C \stackrel{h}{\longrightarrow} D \stackrel{k}{\leftarrow} E$ such that all of the $\mathcal{S}$-relative pullbacks
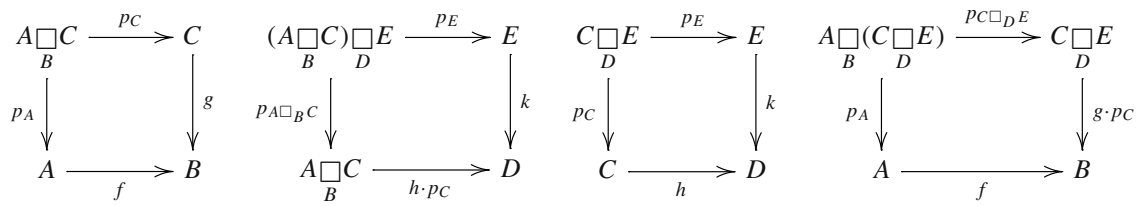

exist. Then $\left(A \square_{B} C\right) \square_{D} E$ and $A \square_{B}\left(C \square_{D} E\right)$ are isomorphic.

(3) Consider morphisms $A \stackrel{f}{\rightarrow} B \stackrel{g}{\leftarrow} C$ such that the first listed $\mathcal{S}$-relative pullback $A \square C$ in part (2) exists. Then the isomorphisms of part (1) and the isomorphism $\left(A \square_{B} B\right) \square_{B} C \rightarrow$ $A \square_{B}\left(B \square_{B} C\right)$ of part (2) satisfy Mac Lane's triangle condition. 
(4) Consider morphisms $A \rightarrow B \leftarrow C \rightarrow D \leftarrow E \rightarrow F \leftarrow G$ such that all of the $\mathcal{S}$ relative pullbacks $A \square_{B} C \square_{D} E \square_{F} G$ with any (hence by part (2) all) possible bracketing exist. Then the isomorphisms of part (2) satisfy Mac Lane's pentagon condition.

To the question of the existence of the $\mathcal{S}$-relative pullbacks in parts (2) and (4) of Proposition 3.6 we shall return in Proposition 4.5.

Proof Part (1) is obvious. For part (2) note that by part (1) of Proposition 3.5 the top row of the commutative diagram

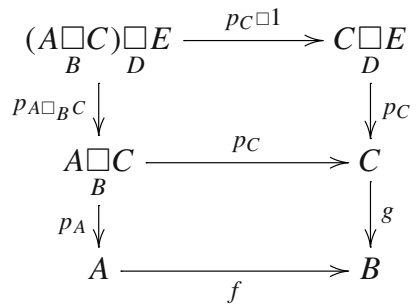

is well-defined. By construction

$$
A \stackrel{p_{A}}{\longleftarrow} A \square_{B} C \stackrel{p_{C}}{\longrightarrow} C \quad \text { and } \quad A \square_{B} C \stackrel{p_{A \square_{B} C}}{\gtrless}(A \underset{B}{\square} C) \underset{D}{\square_{D}} E \stackrel{p_{E}}{\longrightarrow} E
$$

belong to $\mathcal{S}$. Then by properties (PRE) and (POST) of $\mathcal{S}$, respectively, also the spans

$$
\begin{aligned}
& A \ll \stackrel{p_{A}}{\longleftarrow} A \underset{B}{\square} C \stackrel{p_{A \square_{B} C}}{\longleftarrow}(A \underset{B}{\square} C) \underset{D}{\square} \stackrel{p_{A \square_{B} C}}{\longrightarrow} A \underset{B}{\longrightarrow} C \stackrel{p_{C}}{\longrightarrow} C \\
& A<\frac{p_{A}}{\square \square} C \stackrel{p_{A} \square_{B} C}{\longleftarrow}(A \underset{B}{\square} C) \underset{D}{\square} E \underset{p_{C} \square 1}{\longrightarrow} C \underset{D}{\square} E>_{p_{E}} E
\end{aligned}
$$

belong to $\mathcal{S}$. Hence we conclude by the reflection property of $C \square[D$ that

$$
A \stackrel{p_{A}}{\longleftarrow} A \square_{B} C \stackrel{p_{A} \square_{B} C}{\longleftarrow}(A \underset{B}{\longleftarrow} C) \underset{D}{\square} E \stackrel{p_{C} \square 1}{\longrightarrow} C \square_{D} E
$$

belongs to $\mathcal{S}$. With all that information at hand, there is a unique morphism $l$ rendering commutative the first diagram of
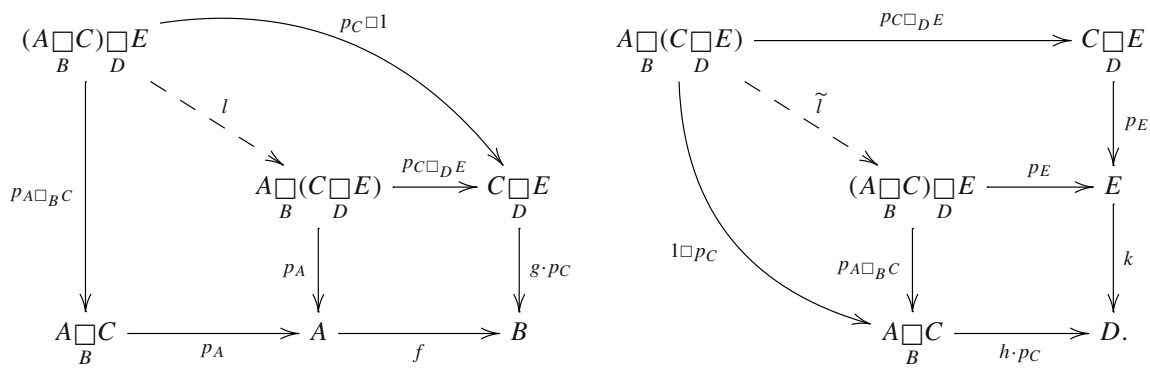
A symmetric reasoning yields a morphism $\tilde{l}$ in the second diagram. Since the right vertical morphisms in the next diagrams are jointly monic, the commutativity of
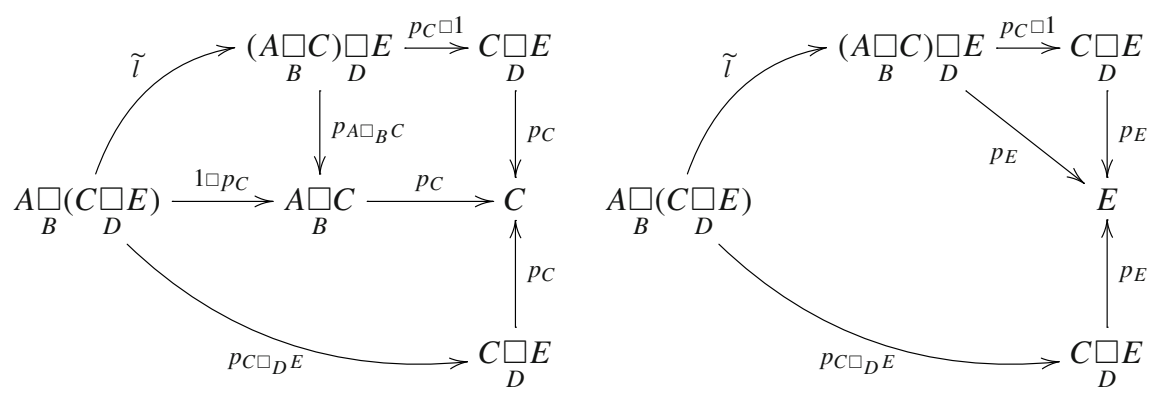

proves $\left(p_{C} \square 1\right) \cdot \tilde{l}=p_{C \square_{D} E}$. This is used to see the commutativity of the second diagram of
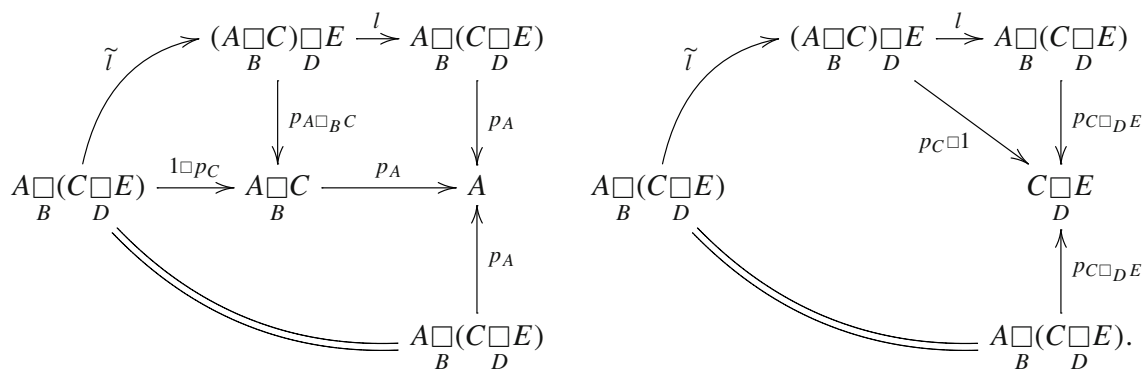

Since their right verticals are jointly monic, the commutativity of these diagrams implies $l \cdot \widetilde{l}=1$. A symmetric reasoning leads to $\widetilde{l} \cdot l=1$ so that $l$ and $\widetilde{l}$ are mutual inverses.

(3) Since $A \stackrel{p_{A}}{\leftarrow} A \underset{B}{\square} \stackrel{p_{C}}{\longrightarrow} C$ are jointly monic, the claim follows by the commutativity of both diagrams below.
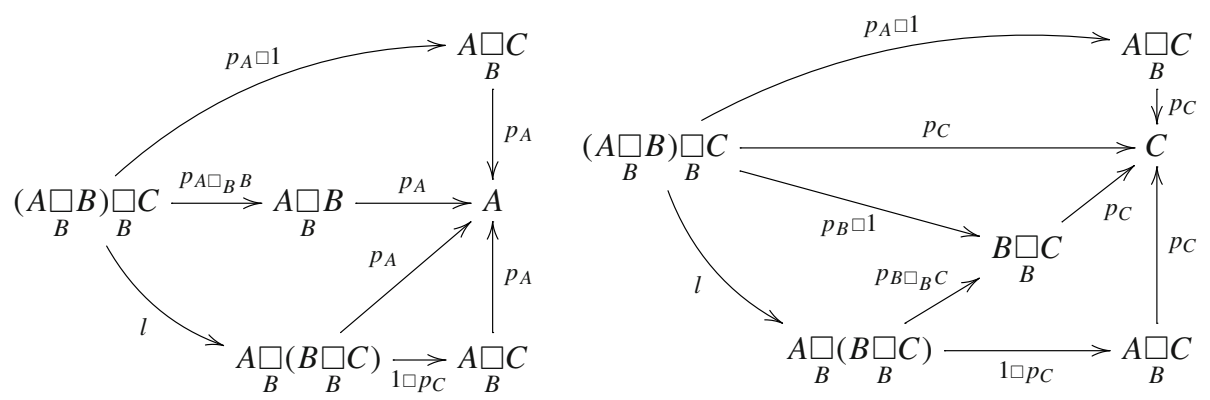

(4) The claim follows by similar standard arguments; using the construction of $l$ and the fact that the morphisms 


$$
\begin{aligned}
& A \underset{B}{\square}(C \underset{D}{\square}(E \underset{F}{\square} G)) \stackrel{p_{A}}{\longrightarrow} A
\end{aligned}
$$

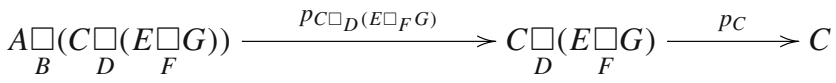

$$
\begin{aligned}
& A \square_{B}\left(C \underset{D}{\square}\left(E \square_{F} G\right)\right) \stackrel{p_{C \square_{D}\left(E \square_{F} G\right)}}{\longrightarrow} C \underset{D}{\square}\left(E \square_{F} G\right) \stackrel{p_{E \square_{F} G}}{\longrightarrow} E \square_{F} G \stackrel{p_{E}}{\longrightarrow} E \\
& A \square_{B}(C \underset{D}{\square}(E \underset{F}{\square} G)) \stackrel{p_{C \square_{D}\left(E \square_{F} G\right)}}{\longrightarrow} C \underset{D}{\square}(E \underset{F}{\square} G) \stackrel{p_{E \square_{F} G}}{\longrightarrow} E \underset{F}{\square} G \stackrel{p_{G}}{\longrightarrow} G
\end{aligned}
$$

are jointly monic.

Since $\mathcal{S}$-relative pullbacks are defined up-to isomorphisms, Proposition 3.6 allows us to pretend that $\square$ is associative and omit the parentheses as well as the isomorphisms $l$ in Proposition 3.6.

Proposition 3.7 For a monoidal admissible class $\mathcal{S}^{\prime}$ of spans in a monoidal category $M$, consider an $\mathcal{S}^{\prime}$-relative pullback

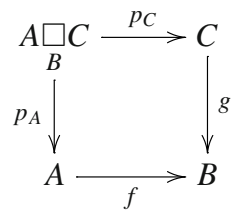

in which $f$ and $g$ are monoid morphisms.

(1) There is a unique monoid structure on $A \square_{B} C$ such that $p_{A}$ and $p_{C}$ are monoid morphisms.

(2) The diagram of (3.7) is a pullback relative to the admissible class $\mathcal{S}$ of spans in the category of monoids in $M$ defined in Example 2.9.

Proof (1) By construction $A \stackrel{p_{A}}{\leftarrow} A \underset{B}{ } C \stackrel{p_{C}}{\rightarrow} C \in \mathcal{S}^{\prime}$ hence by the multiplicativity of $\mathcal{S}^{\prime}$ $A^{2} \stackrel{p_{A} p_{A}}{\longleftarrow}\left(A \square_{B} C\right)^{2} \stackrel{p_{C} p_{C}}{\longrightarrow} C^{2} \in \mathcal{S}^{\prime}$. Then by (POST) $A \stackrel{m}{\leftarrow} A^{2} \stackrel{p_{A} p_{A}}{\longleftarrow}(A \underset{B}{\square} C)^{2} \stackrel{p_{C} p_{C}}{\longrightarrow} C^{2} \stackrel{m}{\longrightarrow} A \in$ $\mathcal{S}^{\prime}$. Hence by the commutativity of the first diagram in
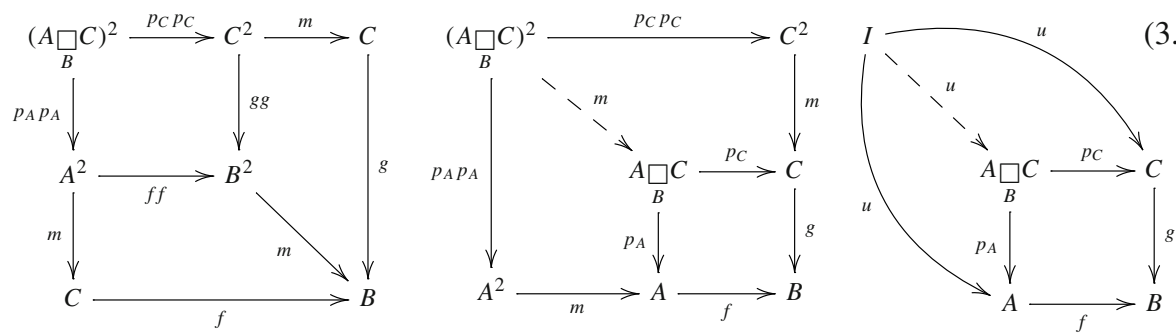

there is a unique filler $m$ for the second diagram of (3.8). By the unitality of $\mathcal{S}^{\prime}$, the span $A \stackrel{u}{\leftarrow} I \stackrel{u}{\longrightarrow} C$ belongs to $\mathcal{S}^{\prime}$. Then by $f \cdot u=u=g \cdot u$, there is a unique filler $u$ for the third diagram of (3.8). By a standard reasoning, associativity and unitality of the monoid $A \square C$ 
follow from the respective properties of $A$ and $C$ making use of the fact that the morphisms $A \stackrel{p_{A}}{<} A \square_{B} C \stackrel{p_{C}}{\longrightarrow} C$ are jointly monic.

(2) Since the span $A \stackrel{p_{A}}{<} A \square_{B} C \stackrel{p_{C}}{\longrightarrow} C$ in $\mathrm{M}$ belongs to $\mathcal{S}^{\prime}$, as a span of monoids it belongs to $\mathcal{S}$ by definition. The square of (3.7) commutes by construction. In order to see its universality, take a span of monoids $A \stackrel{a}{\leftarrow} D \stackrel{c}{\longrightarrow} C$ in $\mathcal{S}$ such that $f \cdot a=g \cdot c$. Then by definition $A \stackrel{a}{\leftarrow} D \stackrel{c}{\longrightarrow} C \in \mathcal{S}^{\prime}$. Since (3.7) is an $\mathcal{S}^{\prime}$-relative pullback in $\mathrm{M}$, there is a unique filler $d$ of the diagram

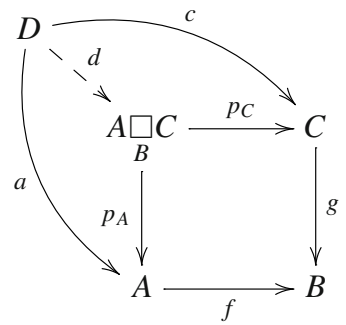

in M. Using again that the morphisms $A \stackrel{p_{A}}{\leftarrow} A \underset{B}{\square} \stackrel{p_{C}}{\longrightarrow} C$ are jointly monic in $\mathrm{M}$, the morphism $d$ is multiplicative by the commutativity of the diagrams
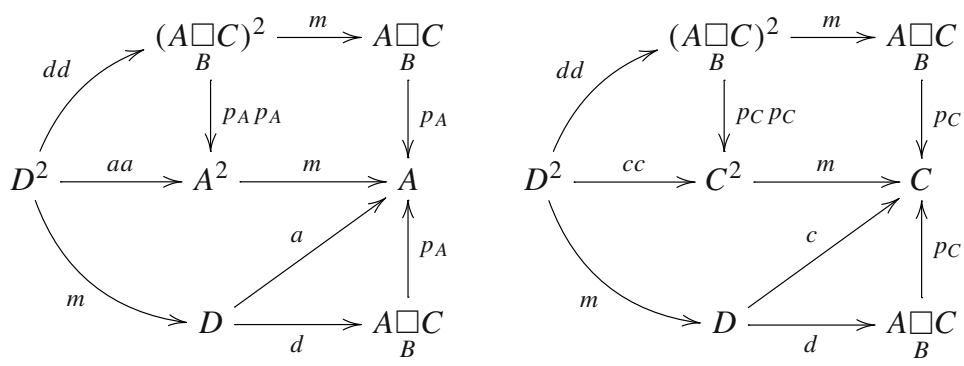

and unital by the commutativity of the diagrams
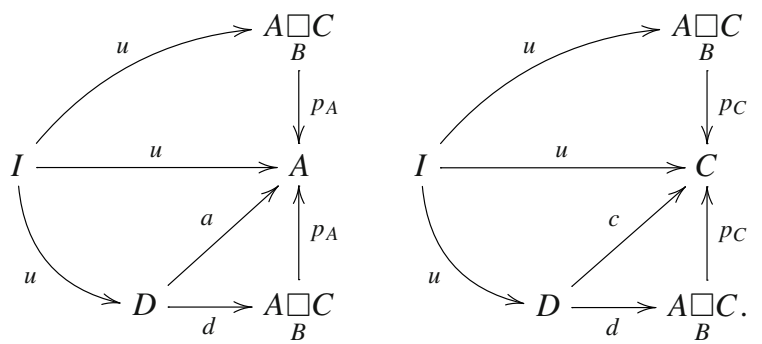

The reflection property is obviously inherited from $M$. 


\section{Relative Categories}

Assumption 4.1 For an admissible class $\mathcal{S}$ of spans in some category $C$ we make the following assumption: whenever a cospan $A \stackrel{f}{\longrightarrow} B \stackrel{g}{\longleftarrow} C$ has legs in $\mathcal{S}$ (see Definition 2.10), there exists their $\mathcal{S}$-relative pullback $A \stackrel{p_{A}}{\longleftarrow} A \underset{B}{\square} \stackrel{p_{C}}{\longrightarrow} C$.

Example 4.2 If $\mathcal{S}$ is the class of all spans in some category C, then Assumption 4.1 reduces to the assumption that pullbacks exist in $C$.

Example 4.3 As in Example 3.3, let $\mathrm{C}$ be the category of comonoids in a braided monoidal category $M$ in which equalizers exist and are preserved by the monoidal product with any object on either side. Then it is proven in Example 3.3 that in $C$ all pullbacks exist relative to the admissible class $\mathcal{S}$ in Example 2.3 of spans in C. Thus in particular Assumption 4.1 holds for this class $\mathcal{S}$.

Example 4.4 Suppose that Assumption 4.1 holds for a monoidal admissible class $\mathcal{S}^{\prime}$ of spans in a monoidal category $\mathrm{M}$. Then it also holds for the admissible class $\mathcal{S}$ of spans in the category of monoids in $\mathrm{M}$ in Example 2.9.

Indeed, if for some monoid morphisms $A \stackrel{f}{\longrightarrow} B \stackrel{g}{\leftarrow} C$ the spans $A=A \stackrel{f}{\longrightarrow} B$ and $B \stackrel{g}{\leftarrow} C=C$ belong to $\mathcal{S}$, then by definition they belong to $\mathcal{S}^{\prime}$ too. Then by Assumption 4.1 there exists their $\mathcal{S}^{\prime}$ relative pullback in $\mathrm{M}$. And it is an $\mathcal{S}$-relative pullback of monoids in $\mathrm{M}$ by Proposition 3.7 .

Proposition 4.5 If Assumption 4.1 holds for some admissible class $\mathcal{S}$ of spans in an arbitrary category $C$ then all of the $\mathcal{S}$-relative pullbacks listed in part (2) of Proposition 3.6 exist provided that the following spans belong to $\mathcal{S}$.

$$
A=A \stackrel{f}{\longrightarrow} B \quad B \stackrel{g}{\leftarrow} C=C \quad C=C \stackrel{h}{\longrightarrow} D \quad D \stackrel{k}{\leftarrow} E=E
$$

Proof Existence of the $\mathcal{S}$-relative pullbacks listed first and third in part (2) of Proposition 3.6 immediately follows by Assumption 4.1. In order to see existence of the $\mathcal{S}$-relative pullback listed second, use first that by the assumption that $C=C \stackrel{h}{\rightarrow} D \in \mathcal{S}$ and by Lemma 3.4 (2) also $A \square C=A \square_{B} C \stackrel{h \cdot p_{C}}{\longrightarrow} D$ is in $\mathcal{S}$. Since $D \stackrel{k}{\leftarrow} E=E$ is in $\mathcal{S}$ by assumption, the existence of the stated $\mathcal{S}$-relative pullback $(A \underset{B}{\square} C) \underset{D}{\square} E$ follows by Assumption 4.1. An analogous reasoning applies to the $\mathcal{S}$-relative pullback $A \underset{B}{\square}(C \underset{D}{\square} E)$ listed last in part (2) of Proposition 3.6.

Corollary 4.6 Consider an admissible class $\mathcal{S}$ of spans in an arbitrary category $C$ for which Assumption 4.1 holds. For any object $B$ in $C$ for which $B=B=B \in \mathcal{S}$, there is a monoidal category whose

objects are spans $B \stackrel{t}{\leftarrow} A \stackrel{s}{\rightarrow} B$ which have their legs in $\mathcal{S}$ (cf. Definition 2.10) morphisms are the morphisms of spans over $B$

$\underline{\text { monoidal product }}$ of $B \stackrel{t}{\leftarrow} A \stackrel{s}{\longrightarrow} B$ and $B \stackrel{t^{\prime}}{\leftarrow} A^{\prime} \stackrel{s^{\prime}}{\rightarrow} B$ is $B \stackrel{t \cdot p_{A}}{\leftarrow} A \square_{B} A^{\prime} \stackrel{s^{\prime} \cdot p_{A^{\prime}}}{\longrightarrow} B$ (its legs are in $\mathcal{S}$ by Lemma 3.4) where $A \square_{B} A^{\prime}$ is the $\mathcal{S}$-relative pullback of $A \stackrel{s}{\rightarrow} B \stackrel{t^{\prime}}{\leftarrow} A^{\prime}$ 
monoidal unit is $B=B=B$.

For any positive integer $n$, we denote by $A^{\square}{ }^{\natural}$ the $n$ 'th monoidal power of the object $B \stackrel{t}{\leftarrow} A \stackrel{s}{\rightarrow} B$. We adopt the convention $A^{\square 0}=B$.

Proof For morphisms of spans, the $\mathcal{S}$-relative pullback in Proposition 3.5 is obviously a morphisms of spans. So in view of Proposition 3.5, Proposition 3.6 and Proposition 4.5, we only need to check the naturality of the unit and associativity constraints in Proposition 3.6. Naturality of the unit constraints-that is, commutativity of
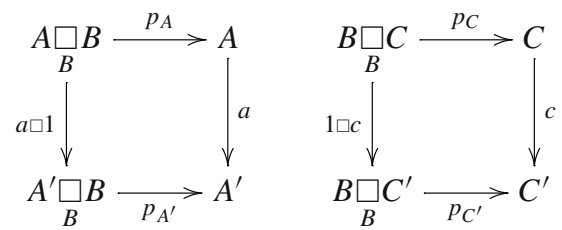

for any morphisms of spans $A \stackrel{a}{\rightarrow} A^{\prime}$ and $C \stackrel{c}{\rightarrow} C^{\prime}$ - holds by construction.

For any morphisms of spans $A \stackrel{a}{\rightarrow} A^{\prime}, C \stackrel{c}{\rightarrow} C^{\prime}$ and $E \stackrel{e}{\rightarrow} E^{\prime}$, let us compose both $(a \square(c \square e)) \cdot l$ and $l \cdot((a \square c) \square e)$ with the jointly monic morphisms

$$
A_{B^{\prime}}^{\square}\left(C_{D^{\prime}}^{\square} E^{\prime}\right) \stackrel{p_{A^{\prime}}}{\longrightarrow} A^{\prime} \quad A^{\prime} \underset{B^{\prime}}{\square}\left(C^{\prime} \underset{D^{\prime}}{\square} E^{\prime}\right) \stackrel{p_{C^{\prime}} \square_{D^{\prime}} E^{\prime}}{\longrightarrow} C^{\prime} \underset{D^{\prime}}{\square} E^{\prime} \stackrel{p_{C^{\prime}}}{\longrightarrow} C^{\prime} \quad A_{B^{\prime}} \underset{D^{\prime}}{\square}\left(C^{\prime} \square \stackrel{p_{C^{\prime}} E_{D^{\prime}} E^{\prime}}{\longrightarrow} C^{\prime} \square{ }_{D^{\prime}} E^{\prime} \stackrel{p_{E^{\prime}}}{\longrightarrow} E^{\prime} .\right.
$$

The resulting pairs of composite morphisms are easily seen to be equal to



respectively. This proves the naturality of the associativity constraint.

It may happen that in some category not only those cospans have pullbacks relative to some class $\mathcal{S}$ of spans whose legs are in $\mathcal{S}$. (Recall from Example 3.3 that in certain categories of comonoids all pullbacks exist relative to the class of spans in Example 2.3). However, the monoidal structure of Corollary 4.6 is available only on the category of those spans whose legs are in $\mathcal{S}$; see Proposition 3.6 (1).

Example 4.7 If $\mathcal{S}$ is the class of all spans in a category $C$ having pullbacks, then Corollary 4.6 describes the monoidal category of spans in $C$ via the usual pullback.

Example 4.8 As in Example 3.3, let $\mathrm{C}$ be the category of comonoids in a braided monoidal category $M$ in which equalizers exist and are preserved by the monoidal product with any object on either side. Then we know from Example 4.3 that Assumption 4.1 holds for the admissible class $\mathcal{S}$ in Example 2.3 of spans in C. For a comonoid $B$ in $\mathrm{M}$ the condition $B=B=B \in \mathcal{S}$ reduces to the cocommutativity of the comonoid $B$. So by Corollary 4.6 the category of spans of comonoids over a cocommutative comonoid $B$ with legs in $\mathcal{S}$ is monoidal via the $B$-cotensor product of (3.6).

Definition 4.9 Consider an admissible class $\mathcal{S}$ of spans in an arbitrary category $C$ for which Assumption 4.1 holds, and an object $B$ in $C$ for which $B=B=B \in \mathcal{S}$. An $\mathcal{S}$-relative 
category with object of objects $B$ is a monoid in the monoidal category of Corollary 4.6. Explicitly, this means the data in

$$
B \underset{t}{\ll} i \longrightarrow A<\quad d \quad A \square_{B} A
$$

subject to the following axioms.

(a) The legs of $B \stackrel{t}{\leftarrow} A \stackrel{s}{\longrightarrow} B$ are in $\mathcal{S}$ (so that the pullback $A \stackrel{p_{1}}{\leftarrow} A \underset{B}{\square} A \stackrel{p_{2}}{\longrightarrow} A$ of $A \stackrel{s}{\longrightarrow} B \stackrel{t}{\leftarrow} A$ relative to the class $\mathcal{S}$ exists).

(b) $i$ is a common section of $s$ and $t$ (that is, $B \underset{t}{\stackrel{s}{\ll} i \geqslant} A$ is a reflexive graph).

(c) $t \cdot d=t \cdot p_{1}$ and $s \cdot d=s \cdot p_{2}$.

(d) $d \cdot(i \square 1)=1=d \cdot(1 \square i)$.

(e) $d \cdot(d \square 1)=d \cdot(1 \square d)$.

Definition 4.10 Consider an admissible class $\mathcal{S}$ of spans in an arbitrary category $C$ for which Assumption 4.1 holds, and objects $B$ and $B^{\prime}$ in $C$ for which $B=B=B$ and $B^{\prime}=B^{\prime}=B^{\prime}$ belong to $\mathcal{S}$. An $\mathcal{S}$-relative functor between $\mathcal{S}$-relative categories as in (4.1) consists of a pair of morphisms $\left(B \stackrel{b}{\rightarrow} B^{\prime}, A \stackrel{a}{\rightarrow} A^{\prime}\right.$ ) which is

(a) a morphism of spans in the sense that $b \cdot s=s^{\prime} \cdot a$ and $b \cdot t=t^{\prime} \cdot a$ (hence by Proposition 3.5 there exists the $\mathcal{S}$-relative pullback morphism $A \underset{B}{\square} \stackrel{a \square a}{\longrightarrow} A^{\prime} \underset{B^{\prime}}{\square} A^{\prime}$ )

(b) it is compatible with the monoid structure in the sense that $a \cdot i=i^{\prime} \cdot b$ and $a \cdot d=d^{\prime} \cdot(a \square a)$.

In Part II, in and after [7, Proposition 3.13], the category of so-called cat ${ }^{1}$-Hopf monoids in a symmetric monoidal category $\mathrm{M}$ in [20] is proven to be a full subcategory of the category of categories relative to the class of spans in Example 2.3 in the category of comonoids in $\mathrm{M}$.

\section{Summary and Outlook}

In this paper pullbacks were introduced relative to a chosen class of spans. On this class we made assumptions which allow for the pullback to define a monoidal structure on the category of spans with their 'legs in this class'. Relative (to the above class of spans) categories were defined as monoids in the so obtained monoidal category. Non-trivial examples are presented in categories of comonoids in braided monoidal categories.

All this is meant to be a preparation for a further analysis to be carried out in [7] and [8]. In these sequel papers we will apply this theory to categories of monoids in symmetric monoidal categories; that is, we consider relative categories of monoids. Those of them for which a canonical family of morphisms are invertible, will be shown to be equivalent to relative crossed modules of monoids (see [7]) and to suitable relative simplicial monoids of Moore length 1 (in [8]).

Again, interesting examples will arise from categories of comonoids in braided monoidal categories; whose monoids are known as bimonoids. Taking the full subcategory of Hopf monoids in a category of bimonoids, some recent results in the literature- $[1,18-20,23,31]-$ will be placed in a broader context. 
Acknowledgements Open access funding provided by MTA Wigner Research Centre for Physics (MTA Wigner FK, MTA EK). The author's interest in the subject was triggered by the excellent workshop 'Modelling Topological Phases of Matter-TQFT, HQFT, premodular and higher categories, Yetter-Drinfeld and crossed modules in disguise' in Leeds UK, 5-8 July 2016. It is a pleasure to thank the organizers, Zoltán Kádár, João Faria Martins, Marcos Calçada and Paul Martin for the experience and a generous invitation. Helpful comments and a list of relevant references from the anonymous referee are highly appreciated. Financial support by the Hungarian National Research, Development and Innovation Office-NKFIH (Grant K124138) is gratefully acknowledged.

Open Access This article is distributed under the terms of the Creative Commons Attribution 4.0 International License (http://creativecommons.org/licenses/by/4.0/), which permits unrestricted use, distribution, and reproduction in any medium, provided you give appropriate credit to the original author(s) and the source, provide a link to the Creative Commons license, and indicate if changes were made.

\section{References}

1. Aguiar, M.: Internal Categories and Quantum Groups, Ph.D. thesis, Cornell University (1997)

2. André, M.: Méthode simpliciale en algèbre homologique et algèbre commutative. Springer LNM 32. Springer, Berlin (1967)

3. Andruskiewitsch, N., Devoto, J.A.: Extensions of Hopf algebras. Algebra i Analiz 7(1), 22-61 (1995). (English version: St. Petersburg Mathematical Journal 7 no. 1 (1995), 17-52)

4. Baez, J.C., Lauda, A.D.: Higher-dimensional algebra V: 2-groups. Theory Appl. Categ. 12(4), $423-491$ (2004)

5. Bántay, P.: Characters of crossed modules and premodular categories. In: Lepowsky, J., McKay, J., Tuite, M.P. (eds.) Moonshine-The First Quarter Century and Beyond. Proceedings of a Workshop on the Moonshine Conjectures and Vertex Algebras. London Math. Soc. LNS 372, pp. 1-12, Cambridge University Press (2010)

6. Baues, H.-J.: Homotopy Type and Homology. Oxford University Press, Oxford (1996)

7. Böhm, G.: Crossed modules of monoids II. Relative crossed modules, preprint arXiv:1803.04124

8. Böhm, G.: Crossed modules of monoids III. Simplicial monoids of Moore length 1, preprint arXiv: 1803.04622

9. Breen, L.: Bitorseurs et cohomologie non abèlienne. In: The Grothendieck Festschrift Vol. I, Progr. Math., vol. 86, pp. 401-476, Birkhäuser Boston, Boston, MA (1990)

10. Breen, L., Messing, W.: Differential geometry of gerbes. Adv. Math. 198(2), 732-846 (2005)

11. Brown, R., Higgins, P.J., Sivera, R.: Nonabelian algebraic topology: Filtered spaces, crossed complexes, cubical homotopy groupoids. EMS Monographs in Math., vol. 15. European Mathematical Society (2010)

12. Brown, R., İçen, İ.: Homotopies and automorphisms of crossed modules of groupoids. Appl. Categ. Struct. 11(2), 185-206 (2003)

13. Brown, R., Spencer, C.B.: $\mathcal{G}$-groupoids, crossed modules and the fundamental groupoid of a topological group. Indag. Math. (Proc.) 79(4), 296-302 (1976)

14. Conduché, D.: Modules croisés généralisés de longueur 2. In: Friedlander, E.M., Karoubi, M. (eds.) Proceedings of the Luminy conference on algebraic KK-theory (Luminy, 1983). J. Pure Appl. Algebra, vol. 34(2-3), pp. 155-178 (1984)

15. Dijkgraaf, R., Witten, E.: Topological gauge theories and group cohomology. Commun. Math. Phys. 129(2), 393-429 (1990)

16. Duskin, J.W.: Preliminary remarks on groups, as quoted in [13]: Unpublished notes, Tulane University (1969)

17. Ellis, G., Steiner, R.: Higher dimensional crossed modules and the homotopy groups of $(n+1)$-ads. J. Pure Appl. Algebra 46(2-3), 117-136 (1987)

18. Emir, K.: The Moore Complex of a Simplicial Cocommutative Hopf Algebra, preprint arXiv: 1905.09620

19. Faria Martins, J.: Crossed modules of Hopf algebras and of associative algebras and two-dimensional holonomy. J. Geom. Phys. 99, 68-110 (2016)

20. Fernández Vilaboa, J.M., López López, M.P., Villanueva Novoa, E.: Cat ${ }^{1}$-Hopf algebras and crossed modules. Commun. Algebra 35(1), 181-191 (2006)

21. García-Martínez, X., Van der Linden, T.: A note on split extensions of bialgebras. Forum Math. 30(5), 1089-1095 (2018)

22. Gran, M., Kadjo, G., Vercruysse, J.: A torsion theory in the category of cocommutative Hopf algebras. Appl. Categ. Struct. 24(3), 269-282 (2016) 
23. Gran, M., Sterck, F., Vercruysse, J.: A semi-abelian extension of a theorem by Takeuchi. J. Pure Appl. Algebra 223, 4171-4190 (2019)

24. Guin-Waléry, D., Loday, J.-L.: Obstructions á l'excision en K-théorie algèbrique. In: Evanston Conference on Algebraic K-theory 1980, Springer LNM 854, pp. 179-216. Springer (1981)

25. Higgins, P.J.: Groups with multiple operators. Proc. Lond. Math. Soc. 3-6(6), 366-416 (1956)

26. Janelidze, G.: Internal crossed modules. Georgian Math. J. 10, 99-114 (2003)

27. Lavendhomme, R., Roisin, J.-R.: Cohomologie non abélienne de structures algébriques. J. Algebra 67, 385-414 (1980)

28. Loday, J.-L.: Spaces with finitely many nontrivial homotopy groups. J. Pure Appl. Algebra 24(2), 179-202 (1982)

29. Mac Lane, S.: Homology. Grundlehren der mathematischen Wissenschaften, vol. 114. Springer, Berlin (1967)

30. Mac Lane, S.: Categories for the Working Mathematician. Graduate Texts in Mathematics, vol. 5, 2nd edn. Springer, Berlin (1978)

31. Majid, S.: Strict quantum 2-groups, preprint arXiv: 1208.6265

32. Martins-Ferreira, N., Montoli, A., Sobral, M.: Semidirect products and crossed modules in monoids with operations. J. Pure Appl. Algebra 217(2), 334-347 (2013)

33. Paoli, S.: Internal categorical structures in homotopical algebra. In: Baez, J.C., May, J.P. (eds.) Towards Higher Categories. IMA Volumes in Mathematics and Its Applications, pp. 85-103. Springer (2009)

34. Patchkoria, A.: Crossed semimodules and Schreier internal categories in the category of monoids. Georgian Math. J. 5(6), 575-581 (1998)

35. Porter, T.: Extensions, crossed modules and internal categories in categories of groups with operations. Proc. Edinb. Math. Soc. 30, 373-381 (1987)

36. Porter, T.: $n$-types of simplicial groups and crossed n-cubes. Topology 32, 5-24 (1993)

37. Porter, T.: The Crossed Menagerie: an introduction to crossed gadgetry and cohomology in algebra and topology. Notes initially prepared for the XVI Encuentro Rioplatense de Álgebra y Geometría Algebraica, in Buenos Aires, 12-15 December 2006, extended for an MSc course (Summer 2007) at Ottawa. https:// ncatlab.org/timporter/show/crossed+menagerie

38. Porter, T.: Topological quantum field theories from homotopy n-types. J. Lond. Math. Soc. 58(3), 723-732 (1998)

39. Porter, T.: Homotopy quantum field theories meets the crossed menagerie: an introduction to HQFTs and their relationship with things simplicial and with lots of crossed gadgetry. Notes prepared for the Workshop and School on Higher Gauge Theory, TQFT and Quantum Gravity Lisbon, February, 2011. https://ncatlab.org/timporter/show/HQFTs+meet+the+Crossed+Menagerie

40. Porter, T., Turarev, V.G.: Formal homotopy quantum field theories, I: formal maps and crossed C-algebras. J. Homotopy Relat. Struct. 3, 113-159 (2008)

41. Quillen, D.G.: Homotopical Algebra. Springer LNM 53. Springer, Berlin (1967)

42. Van der Linden, T.: Homology and homotopy in semi-Abelian categories, Ph.D. thesis, Vrije Universiteit Brussel (2006)

43. Whitehead, J.H.C.: Combinatorial homotopy I. Bull. Am. Math. Soc. 55, 213-245 (1949)

44. Whitehead, J.H.C.: Combinatorial homotopy II. Bull. Am. Math. Soc. 55, 453-496 (1949)

45. Yetter, D.N.: Topological quantum field theories associated to finite groups and crossed G-sets. J. Knot Theory Ramif. 1, 1-20 (1992)

46. Yetter, D.N.: TQFT's from homotopy 2-types. J. Knot Theory Ramif. 2, 113-123 (1993)

Publisher's Note Springer Nature remains neutral with regard to jurisdictional claims in published maps and institutional affiliations. 